\title{
Article
}

\section{Anti-CTLA-4 therapy for malignant mesothelioma}

Guazzelli, Alice, Bakker, Emyr, Krstic-Demonacos, Marija, Lisanti, Michael P, Sotgia, Federica and Mutti, Luciano

Available at http://clok.uclan.ac.uk/21179/

Guazzelli, Alice, Bakker, Emyr ORCID: 0000-0002-0091-1029, Krstic-

Demonacos, Marija, Lisanti, Michael P, Sotgia, Federica and Mutti, Luciano (2017) Anti-CTLA-4 therapy for malignant mesothelioma. Immunotherapy, 9 (3). pp. 273-280. ISSN 1750-743X

It is advisable to refer to the publisher's version if you intend to cite from the work. http://dx.doi.org/10.2217/imt-2016-0123

For more information about UCLan's research in this area go to http://www.uclan.ac.uk/researchgroups/ and search for < name of research Group>.

For information about Research generally at UCLan please go to http://www.uclan.ac.uk/research/

All outputs in CLoK are protected by Intellectual Property Rights law, including Copyright law. Copyright, IPR and Moral Rights for the works on this site are retained by the individual authors and/or other copyright owners. Terms and conditions for use of this material are defined in the policies page.

\section{CLoK}

Central Lancashire online Knowledge www.clok.uclan.ac.uk

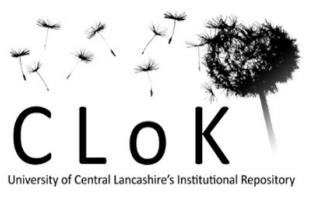




\section{AUTHORS' FINAL VERSION}

ARTICLE PUBLISHED IN FUTURE MEDICINE IMMUNOTHERAPY:

Guazzelli, A., Bakker, E., Krstic-Demonacos, M., Lisanti, M. P., Sotgia, F. \& Mutti, L. 2017. AntiCTLA-4 therapy for malignant mesothelioma. Immunotherapy, 9, 273-280. 


\section{Abstract}

Immunotherapy is an emerging therapeutic strategy with a promising clinical outcome in some solid tumours, particularly metastatic melanoma. One approach to immunotherapy is immune checkpoint inhibitors, such as blockage of cytotoxic T-lymphocyte antigen-4 (CTLA-4) and programmed death1 (PD-1) and/or programmed death-ligand 1 (PD-L1).

This special report aims to describe the state of clinical trials of tremelimumab in patients with unresectable Malignant Mesothelioma (MM) in particular with regard to the clinical efficacy, safety and tolerability. Criticism and perspective of this treatment are also discussed.

Biological and clinical considerations rule out the use of tremelimumab as single agent for MM and, more in general, the use of immune checkpoint inhibitors for MM is still largely questionable and not supported by evidences.

Keywords: Malignant mesothelioma, immunotherapy, anti-CLTA-4 


\section{Introduction}

Malignant Mesothelioma (MM) is an aggressive tumour of mesothelial cells that may be pleural (>80\%), pericardial, peritoneal or vaginal/testicular and is associated with asbestos exposure [1, 2]. Although rare, MM incidence is increasing, especially in industrialized nations where asbestos is not banned [3] and the incidence is expected to peak between 2015-2030 [4]. Simian virus 40, radiation, erionite and genetic susceptibility may contribute to MM development $[5,6]$.

MM has poor prognosis (median survival of 9-12 months) and the majority of patients are diagnosed in an advanced stage [1]. The lack of effective diagnostic tools for early detection and a lack of validated circulating biomarkers complicate diagnosis [7-11]. Combination chemotherapy with antifolate/cisplatin is the standard first-line treatment, which prolongs median survival by just 2.8 months [12]. Second-line treatments are not still established. The need to discover new druggable targets is urgently required to overcome the poor prognosis of MM. Immunotherapy, in particular immune checkpoint blockade, is currently one of the most explored and attractive therapeutic approaches in oncology [13]. Immune checkpoint therapy, using blocking antibodies to CTLA-4 and PD-1/ PD-L1 aim to aid the immune system in the elimination of cancer cells [14]. In this special report we will discuss and comment on current clinical studies of anti-CTLA-4 therapy alone and in combination with PD1/PD-L1 inhibitors as double-checkpoint therapy in patients with MM.

\section{Immunotherapy}

Immunotherapy is an emerging therapy that has acquired clinical interest in cancer treatment [15]. The use of monoclonal antibodies (mAbs) in cancer has shown good promise. MAbs interfere with a single target molecule, with high selectivity due to their specificity [16]. Therefore, the innovative principle of immunotherapy is to not target cancer cells but "educate" the immune system to recognise neoplastic cells. Theoretically, immunotherapy could provide long-term disease control and improve survival by limiting tumour expansion [17].

Ipilimumab and tremelimumab (CP-675,206), both mAbs against CTLA-4, are the main clinically developed immunotherapy drugs and have entered advanced clinical trials for several cancers. Both are fully human novel mAbs, which are able to interrupt co-inhibitory pathways, promoting T-cell activation and tumour-specific cytotoxicity (Fig. 1) [18]. Ipilimumab is an immunoglobulin IgG1 isotype and has a half-life of 12 to 14 days. Tremelimumab is a non-complement-fixing IgG2 isotype, 
which causes less antibody-dependent cellular toxicity than IgG1 and has a half-life of 22 days, thus making tremelimumab a potentially better candidate than ipilimumab [19]. Ipilimumab has been approved by Food and Drug administration (FDA) in unreseactable advanced stage of melanoma [20]. However, tremelimumab remains under investigation at different doses as monotherapy or in combinations in different tumours, including MM.

After the success of ipilumumab in cutaneous melanoma patients, additional checkpoint-blocking mAbs have been developed against PD-1 and its ligand CD274 (PD-L1). Nivolumab (Opdivo ${ }^{\circledR}$, BMS936558, MDX1106) and pembrolizumab (Keytruda ${ }^{\circledR}$ MK-3475, lambrolizumab) represent the first generation of humanized anti-PD-1 antibodies and currently are the main PD-1/PD-L1 blockade approved by the FDA to treat advanced melanoma [21].

\section{Immunotherapy mechanisms of action and the rationale behind the preclinical studies}

CTLA-4 (CD152) is a regulatory molecule, involved in the negative regulation of T-cells [22, 23]. CTLA-4 receptor and the co-stimulatory receptor CD28 share identical ligands: CD80 (B7.1) and CD86 (B7.2) and have the role of interfering the CD28-B7 axis, resulting in a reduction of T-cell proliferation and a decrease in interleukin-2 (IL-2) production [24]. Effector T-cells regulate CTLA4 expression on the plasma membrane after induction of the co-stimulatory interaction between CD28 and B7 on APCs (Fig. 1). Treg cells, however, express CTLA-4 constitutively to maintain their suppressive function [25]. In cancer, it has been shown that the predominance of co-inhibitory pathways influences the feature of tumour evasion from immune system control [26, 27]. Therefore, the discovery of this negative signalling receptor and its critical role in tumour-induced immune tolerance by in vitro studies and preclinical models has allowed the development of a new class of immunotherapeutic drugs [28-30]. Indeed, the crucial function of CTLA4 for negatively regulating T-cell activation has been demonstrated by the fatal systemic immune hyperactivation phenotype of Ctla4-knockout mice, providing the rationale for anti-CTLA-4 therapy [28, 29].

Another study using mesothelioma-bearing mice showed that treatment with anti-CTLA-4 antibody between cisplatin cycles reduced tumour growth and increased survival [30]. The survival and reduced tumour growth was related to improved antitumor immune system responses, including an increase of the total number of T-cells, $\mathrm{CD} 4^{+} / \mathrm{CD}^{+} \mathrm{T}$-cells infiltrating into the tumour, and upregulation of IL-2, promoting T-cell activation. These positive preclinical outcomes lead to clinical studies, highlighting CTLA-4 as the first immune checkpoint target for immunotherapy [31]. 
PD-1 is another immune checkpoint marker expressed on T-cell surfaces, which regulates inhibitory signals by its ligand PD-L1 and together with CLTA-4 represents the main promising cancer immunotherapeutic targets. Preclinical studies showed PD-L1 to be present on murine mesothelioma cells in vivo [32]. PD-L1 overexpression has been detected in response to interferon (IFN)- $\gamma$ and Tcells in tumour-draining lymph nodes [32]. PD-L1 activation has been reported in mesothelioma specimens and is associated with worse prognosis [33-35]. From these results PD-L1 may represent a potential target for novel therapies in MM.

\section{Interaction between the immune system and cancer}

Cancer development is a multistep process in which different systems and molecular components are actively responsible for malignant properties [36]. Understanding the molecular mechanisms involved in the cancer microenvironment, in particular the role of stromal and inflammatory cells, is crucial to identify new targets as diagnostic biomarkers or to develop possible novel therapeutics. One particularly important component is the immune system [37]. This system is very tightly regulated by several cellular populations to activate both innate and adaptive immunity against pathogens. As they are an important component of adaptive immunity, T-cells require complex interactions of signals to be activated [38]. The main stimulatory regulation is mediated by signals between T-cell receptor (TCR) and peptide-major histocompatibility complexes (MHCs) on antigenpresenting cells (APCs). The interaction is amplified by an additional balance between costimulatory/co-inhibitory signals which play a central function in maintaining a sufficient immune response against pathogenic antigens and limited response against self-antigens, guaranteeing an immunologic homeostasis [38, 39].

Therefore, in normal conditions, the immune checkpoint receptors, such as CTLA-4, PD-1 and myeloid-derived suppressor cell (MDSC) interact with their ligands (B7, PD-L1) on various cells types, including APCs, regulatory $\mathrm{T}$-cells $\left(\mathrm{T}_{\text {regs }}\right)$ resulting in reduced $\mathrm{T}$-cell proliferation and functional activity. In the tumour microenvironment, immunologic tolerance has recently been shown to have major implications for the ability of tumours to survive and, in addition, immunosuppressive elements such as CTLA-4, and MDSC are implicated in this mechanism through a variety of pathways. It has been shown that in cancer, immune checkpoints (which exert a negative effect on the immune system) are consistently activated, which leads to a loss of balance between costimulatory and co-inhibitory pathways, thus increasing tumour evasion [40]. 


\section{Immune response to $\mathrm{MM}$}

Research into the role of the immune response in MM has been carried out, with the research highlighting the importance of the immune system in the development and promotion of MM. Once the asbestos fibres are inhaled, macrophages unsuccessfully attempt to metabolize and destroy this foreign body, leading to chronic inflammation [41]. Furthermore, reports indicate that survival may be increased by elevated numbers of CD8+ tumour-infiltrating lymphocytes [42]. $\mathrm{T}_{\text {regs, }}$ however, may infiltrate the tumour microenvironment and influence tumour growth. It has been shown that depletion of $\mathrm{T}_{\text {regs }}$ increases survival [43].

Features of immunogenicity in MM have been shown by studies that demonstrate that MM cells can act as Antigen Presenting Cells [44] and express Cancer Testis Antigens (CTA) noted as potential target for immunotherapy [45]. On the contrary MM cells interfere negatively with the immune activities dependent on IL-2 [46] and suppress the switching of T-cells towards a TH1 profile. Transforming growth factor-beta released by Purified Protein Derivative (PPD)-presenting malignant mesothelioma cells inhibits interferon-gamma synthesis by an anti-PPD CD4+ T-cell clone [47].

However, the first clinical studies related to these molecules have been disappointing [46, 48]. Therefore, the attention was focused on immunotherapeutic strategies using monoclonal antibodies (mAbs) that target receptors of immune cell surface or molecules expressed on tumour cells which regulate the anti-tumour activity of T-cells. Among these molecules, CTLA-4 and B7-H3 have been investigated in MM as immunotherapy [49] and have provided a rationale for their therapeutic use for this tumour.

\section{Clinical studies of tremelimumab in unresectable MM patients}

So far, a second-line therapy for MM is not established and there are no treatments that significantly prolong survival rate. The urgent need to find both high efficacy and well-tolerated therapy for refractory MM patients and the potential application of anti-CTLA-4 antibodies has led to the design of an experimental study termed MESOT-TREM-2008 (NCT01649024) (Table 1) [50]. This study is an open-label, single-arm phase 2 trial where the clinical aspects and immunological activity of tremelimumab were evaluated in advanced malignant mesothelioma patients who failed the first-line 
treatment based on platinum-based regimen [50]. Twenty-nine highly selected patients (limited disease and very good performance status) were enrolled and received a dose of $15 \mathrm{mg} / \mathrm{kg}$ intravenously once every 90 days.

The primary endpoint was the proportion of treated patients who reached a complete or partial objective response according to the Response Evaluation Criteria in Solid Tumors (RECIST) for peritoneal malignant mesothelioma and modified RECIST criteria for MM. The secondary endpoint was to include the proportion of treated patients with disease control, progression-free survival (PFS), overall survival (OS), safety, and changes in lymphocyte phenotype induced by tremelimumab. Since only two patients (7\%) achieved a durable partial response (one lasting 6 months and the other one lasting 18 months), the primary endpoint was not reached. Nine patients (31\%) showed disease control, median PFS was 6.2 months and OS was 10.7 months. Serious side effects $\left(3^{\circ}-4^{\circ}\right.$ grade with one death due to gastrointestinal toxicity) were observed in four (14\%) patients, with one demonstrating hepatic and pancreatic toxicity, and two developing gastrointestinal adverse events. The patient who achieved long-lasting partial response developed a Guillain-Barré-like syndrome. The persistent disease control and the long-lasting partial response together with an initial progressive disease are considered typical features of CTLA-4 treatment, as already reported in metastatic melanoma patients treated with ipilimumab [51]. Since these are non-typical patterns of clinical response, it is necessary to carefully evaluate these patterns before interrupting CTLA-4 because the clinical benefits may be delayed.

These findings lead to a redefinition of the response criteria for immune checkpoint therapies, defined immune-related response criteria (irRC) [52]. The main criteria, which distinct irRC from RECIST are requirement of confirmation of progression disease (PD) with two subsequent scans at least 4 weeks later, and new lesions are not necessary linked to PD but they need to be included in the total tumour burden (Table 2) [53]. Moreover, an increased number of circulating $\mathrm{CD} 4{ }^{+} \mathrm{ICOS}^{+} \mathrm{T}$ lymphocytes, ICOS, expressed on the cell surface of activated T-cells, plays a role in T-cell expansion and survival, was associated with improved survival as reported in studies of ipilimumab or tremelimumab in melanoma and breast cancer [54, 55]. These findings suggest that circulating $\mathrm{CD}^{+}{ }^{+} \mathrm{ICOS}^{+} \mathrm{T}$ lymphocytes may be a general tool to guide the use of anit-CTLA-4 mAbs.

Another phase 2 study for MM, called MESOTREM-2012 (NCT01655888) has explored a more intensive dosing schedule (Table 1). MESOTREM-2012 is an open-label, single arm, phase 2 trial 
for patients with unresectable, advanced MM (pleural or peritoneal) and progression disease after a platinum-based treatment regimen [56]. Tremelimumab was administrated for 1 hour intravenously at a dose of $10 \mathrm{mg} / \mathrm{kg}$ every 4 weeks for six doses, followed by maintenance dosing every 12 weeks. In this study, the primary endpoint was the proportion of treated patients who reached a complete or partial immune-related objective response according to the irRC criteria. The secondary endpoint was to include the proportion of treated patients with immune-related disease control, immune-related progression-free survival, OS, safety, and changes in lymphocyte phenotype induced by tremelimumab (see the comments on irRC in the "perspective" section for further details).

The primary endpoint was reached and four (14\%) patients achieved an immune-related partial response, fifteen (52\%) treated patients had a disease control (median of 10.9 months) and the median immune-related PFS was 6.2 months, whilst 11.3 months was the median overall survival. A modest increase of side effects (grade $1^{\circ}$ and $2^{\circ}$ ) was observed compared to the previous study; two patients had grade 3 hepatic or gastrointestinal toxic effects. From this study, it was observed that the best population of T-cell in response to CTLA-4 blockade is ICOS-positive T-cell subset, which may represent a more accurate pharmacodynamics marker to predict better survival. These findings are currently under investigation in another randomised, double-blind, placebo-controlled, phase $2 \mathrm{~b}$ trial study (NCT01843374) (Table 3).

Recently, it has been announced by AstraZeneca and MedImmune that tremelimumab as monotherapy does not improve survival and the primary endpoint is not reached (D4880C00003; NCT01843374) [57]. Further data regarding DETERMINE was presented at ASCO 2016, detailing that 571 patients were enrolled and that $81 \%$ had died throughout the duration of the study [58]. There was no statistically significant difference between treated patients and placebo patients, and side effects such as diarrhoea, loss of appetite, and development of rashes were seen at higher rates in treated patients than in placebo treatments [58]. This indicates a need for further research into the use of tremelimumab as a monotherapy as well as use in combination therapy to potentially achieve better clinical outcomes.

\section{Combination Immunotherapy}

It is hypothesised that combination immunotherapy could yield better clinical outcomes than monotherapy. Nivolumab and pembrolizumab, two PD-1 inhibitors, have been approved to treat patients with advanced and metastatic melanoma, as well as refractory non-small cell lung cancer [59]. Their use in mesothelioma is much less well-established, however there are some clinical trials 
that are currently underway or in recruiting. One trial (KEYNOTE-028) detailed preliminary findings on the use of pembrolizumab in MM and demonstrated that the drug was well-tolerated and had some anti-tumour effect [60]. However, for immunotherapy in general and in particular for immunotherapy for MM, much more research is needed, particularly due to failure of some studies to meet the primary endpoint [57]. The safety profile of combined immunotherapy is another aspect worth clinical assessment.

There have been several clinical studies combining anti-PD-1 and anti-CTLA-4 agents, such as one in advanced melanoma which identified that the combination therapy nivolumab and ipilimumab lead to an objective response of $53 \%$, with up to $80 \%$ in tumour mass [61]. Another trial, using untreated patients with metastatic melanoma, demonstrated that nivolumab was more effective than ipilimumab (as was combination of nivolumab and ipilimumab against ipilimumab alone), and that for PD-1negative patients combination therapy was more effective than monotherapy [62]. The combination of these targets has been approved for use in patients with BRAF wild-type metastatic or unresectable melanoma [59]. Again, the use of the combination therapy is less well-established in MM than for other cancer types, likely due to the slower development for the drugs as a monotherapy. However, anti-CTLA4 and anti-PD-L1 trials have been reported to be underway [63], and a study design for tremelimumab and durvalumab combination for MM was presented at ASCO 2016 (NIBIT-MESO1) [64]. This study is in early stages and according to the ClinicalTrials.gov page it is still recruiting.

Ultimately immunotherapy, whether as a monotherapy or in combination, is still in its very early stages for mesothelioma. The recent setback of tremelimumab trial not meeting its study criteria [57] and the early stage of clinical trials signify the amount of work still needed in this area.

\section{Key issues and future perspectives}

Even though the potential of a future use of tremelimumab for MM looks has been largely jeopardized by the latest clinical outcome (which included some cases of very severe toxicity and a lack of effect on survival), some more general comments should be considered before proceeding with immune checkpoint inhibitors for MM.

-Biological comments: recent studies on stroma and the microenvironment provide significant evidence which may suggest a potential therapeutic use of check point inhibitors for MM. Among 
other factors, T-cell exclusion looks to be likely to confer resistance of MM to this type of therapy [65].

-Clinical comments: so far only MM patients who are very fit and with limited disease have been recruited into checkpoint inhibitors trials; irRC as a response criteria are very arguable and unlikely to be reliable for this tumour whose thoracic extension and actual shrinkage after treatment are very hard to measure. Patient stratification is a very relevant issue that should be properly addressed but is still at early stages; as the study population is getting larger, our knowledge on acute and chronic toxicity is unravelling more side effects that should be now considered. Lastly, though probably the most important aspect, is the real impact on survival of these treatment. Other than some anecdotal very good responses, no positive impact on survival has been achieved with these treatments so far.

-Cost/benefit: all the above comments cannot be considered without bearing in mind the financial cost that the use of these drugs implies and that, at the moment, such cost cannot be justified by the clinical results.

-Future indication: when the toxicity results reach acceptable levels, the effect on survival of combined checkpoint inhibitors and of checkpoint inhibitors along with chemotherapy are worth investigating. Addressing checkpoint inhibition and the microenvironment is clearly a further fascinating, although still speculative, option. Eventually an adjuvant role of these treatments could be also cautiously hypothesized. All of the above biological and clinical comments are clearly in place also for these potential approaches.

Financial disclosure

Nonfinancial— No relevant nonfinancial relationship exists. 
Executive summary

- It has been shown that in cancer, immune checkpoints (which exert a negative effect on the immune system) are consistently activated, which leads to a loss of balance between co-stimulatory and co-inhibitory pathways, thus increasing tumour evasion.

- The role of immune responses has been deeply investigated in $\mathrm{MMe}$, highlighting the importance of the immune system in developing and promoting MMe.

- CTLA-4 is an immune checkpoint receptor which regulates inhibitory signals to guarantee an immunological homeostasis.

- Blockage of the CTLA-4 receptor allows T-cell activation and tumour-specific T-cells to exert cytotoxic effects on tumour cells.

- Tremelimumab is a fully human monoclonal antibody used in clinical trials for unresectable MMe patients.

- Conclusion: biological and clinical considerations may rule out the use of Tremelimumab as single agent for MMe and the use of immune checkpoint inhibitors for MMe is still largely questionable and not supported by current evidence.

A

\section{Co-stimulatiory signals}

- T-cell activation

- Cytokine produtcion

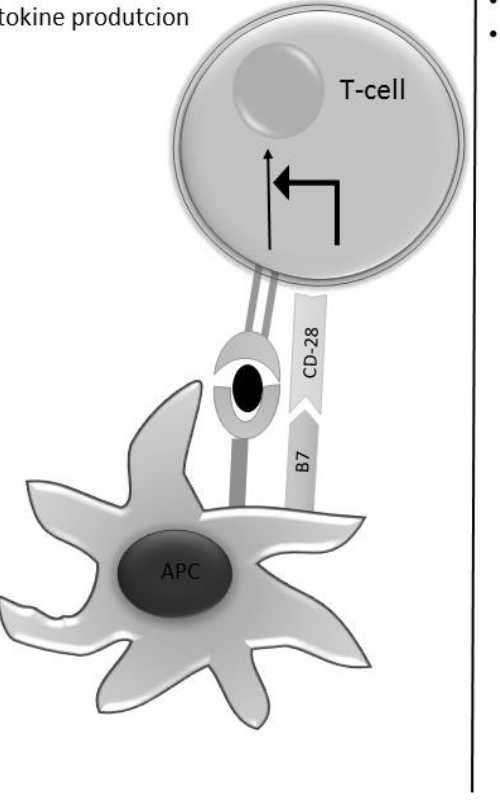

Co-inhibitory signals

Self- tollerance

No T-cell activation
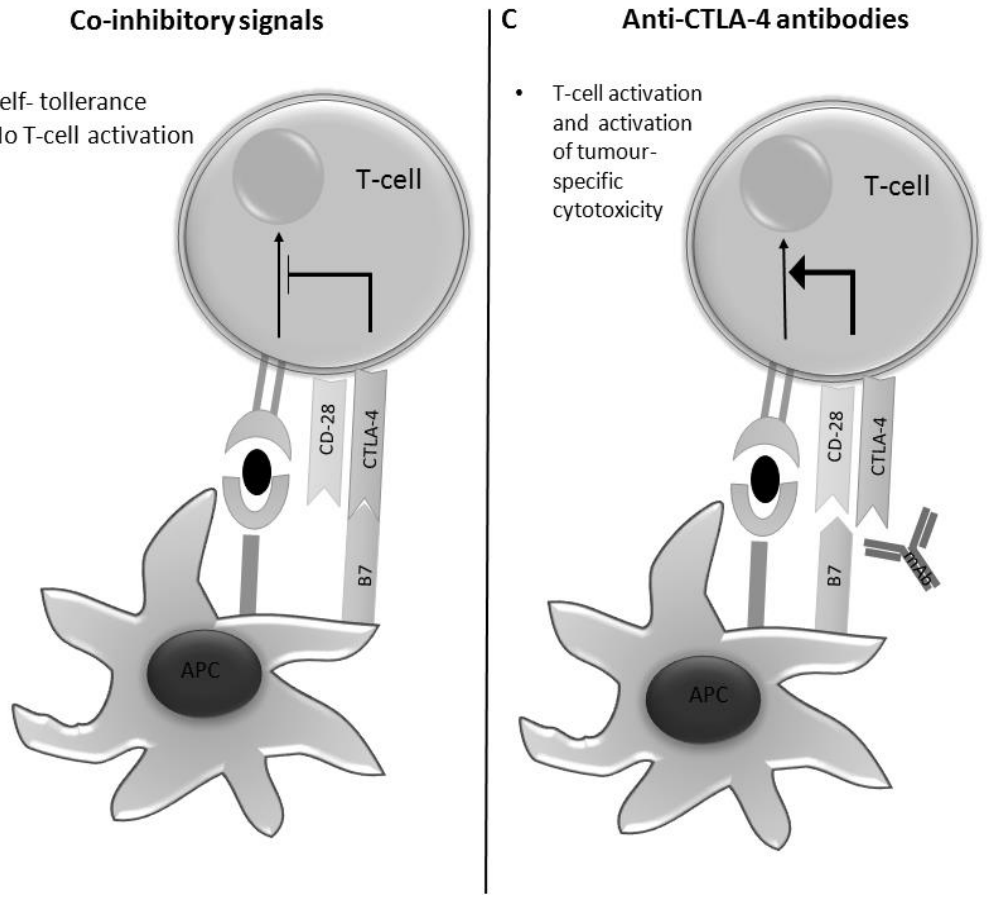

Figure 1. CTLA-4-B7 axis is a co-inhibitor signal for T-cell activation. Effector T-cell activation is mediated by a main interaction between TCR and MHC which is amplified by a co-stimulatory signal (B7/CD-28 binding) (A). Self-tolerance and immunologic homeostasis is regulated by a negative signal between CTLA-4 with B7 in normal cells (B). AntiCTLA-4 antibodies inhibit CTLA-4 activity (C). 
Table 1. Clinical trials with tremelimumab in advanced malignant mesothelioma patients.

\begin{tabular}{|c|c|c|c|}
\hline Study & Clinical ID & Aim & $\begin{array}{l}\mathrm{N}^{\circ} \text { of enrolled } \\
\text { patients }\end{array}$ \\
\hline MESOT-TREM-2008 & NCT01649024 & $\begin{array}{l}\text { The immunologic and clinical activity of } \\
\text { Tremelimumab as second line treatment }\end{array}$ & 29 \\
\hline MESOT-TREM-2012 & NCT01655888 & $\begin{array}{l}\text { The efficacy of a more intensive schedule of } \\
\text { treatment with Tremelimumab as second line } \\
\text { treatment }\end{array}$ & 29 \\
\hline D4880C00003 & NCT01843374 & $\begin{array}{l}\text { Randomized, double-blind, parallel-group study } \\
\text { (Tremelimumab versus placebo as second/third } \\
\text { line) }\end{array}$ & 564 \\
\hline
\end{tabular}

Table 2. Overall response of immune related response criteria (irRC) Information taken from Wolchok and colleagues [52].

\begin{tabular}{|c|l|}
\hline $\begin{array}{c}\text { Complete Response } \\
\text { (CR) }\end{array}$ & $\begin{array}{l}\text { immune related response criteria (irRC) } \\
\text { Disappearance of all lesions in two consecutive observations not } \\
\text { less than four weeks apart }\end{array}$ \\
\hline Partial response (PR) & $\begin{array}{l}\text { A } \geq 50 \% \text { decrease in tumour burden compared with baseline in two } \\
\text { observations at least four weeks } \\
\text { Apart }\end{array}$ \\
\hline $\begin{array}{c}\text { Stable Disease (SD) } \\
\text { Progression Disease } \\
\text { (PD) }\end{array}$ & $\begin{array}{l}\text { A } 50 \% \text { decrease in tumour burden compared with baseline cannot } \\
\text { be established nor } 25 \% \text { increase compared with nadir }\end{array}$ \\
\hline $\begin{array}{l}\text { At least 25\% increase in tumour burden compared with nadir (at } \\
\text { any single time point) in two consecutive observations at least four } \\
\text { weeks apart }\end{array}$
\end{tabular}




\section{Bibliography}

1. Robinson BWS, Musk AW, Lake RA. Malignant mesothelioma. Lancet 366(9483), 397-408 (2005).

2. Ismail-Khan R, Robinson LA, Williams CC, Jr., Garrett CR, Bepler G, Simon GR. Malignant pleural mesothelioma: a comprehensive review. Cancer control : journal of the Moffitt Cancer Center 13(4), 255-263 (2006).

3. Robinson BW, Lake RA. Advances in malignant mesothelioma. The New England journal of medicine 353(15), 1591-1603 (2005).

4. Neumann V, Loseke S, Nowak D, Herth FJ, Tannapfel A. Malignant pleural mesothelioma: incidence, etiology, diagnosis, treatment, and occupational health. Deutsches Arzteblatt international 110(18), 319-326 (2013).

5. Carbone M, Kratzke RA, Testa JR. The pathogenesis of mesothelioma. Seminars in oncology 29(1), 217 (2002)

6. Testa JR, Cheung M, Pei J et al. Germline BAP1 mutations predispose to malignant mesothelioma. Nature genetics 43(10), 1022-1025 (2011).

7. Sekido Y. Molecular pathogenesis of malignant mesothelioma. Carcinogenesis 34(7), 1413-1419 (2013).

8. Napolitano A, Antoine DJ, Pellegrini L et al. HMGB1 and Its Hyperacetylated Isoform are Sensitive and Specific Serum Biomarkers to Detect Asbestos Exposure and to Identify Mesothelioma Patients. Clin Cancer Res 22(12), 3087-3096 (2016).

9. Hollevoet K, Sharon E. Fibulin-3 as a Biomarker for Pleural Mesothelioma. New Engl J Med 368(2), 189-189 (2013).

10. Cristaudo A, Foddis R, Bonotti A et al. Comparison between plasma and serum osteopontin levels: usefulness in diagnosis of epithelial malignant pleural mesothelioma. Int J Biol Marker 25(3), 164-170 (2010).

11. Cristaudo A, Foddis R, Vivaldi A et al. Clinical significance of serum mesothelin in patients with mesothelioma and lung cancer. Clin Cancer Res 13(17), 5076-5081 (2007).

12. Vogelzang NJ, Rusthoven JJ, Symanowski J et al. Phase III study of pemetrexed in combination with cisplatin versus cisplatin alone in patients with malignant pleural mesothelioma. Journal of clinical oncology : official journal of the American Society of Clinical Oncology 21(14), 2636-2644 (2003).

13. Kyi C, Postow MA. Checkpoint blocking antibodies in cancer immunotherapy. FEBS letters 588(2), 368-376 (2014).

14. Yang Y. Cancer immunotherapy: harnessing the immune system to battle cancer. The Journal of clinical investigation 125(9), 3335-3337 (2015). 
15. Kirkwood JM, Butterfield LH, Tarhini AA, Zarour H, Kalinski P, Ferrone S. Immunotherapy of cancer in 2012. CA: a cancer journal for clinicians 62(5), 309-335 (2012).

16. Neves H, Kwok HF. Recent advances in the field of anti-cancer immunotherapy. BBA clinical $3280-$ 288 (2015).

17. Hanoteau A, Moser M. Chemotherapy and immunotherapy: A close interplay to fight cancer? Oncoimmunology 5(7), e1190061 (2016).

18. Blank CU, Enk A. Therapeutic use of anti-CTLA-4 antibodies. Int Immunol 27(1), 3-10 (2015).

19. Ascierto PA, Marincola FM, Ribas A. Anti-CTLA4 monoclonal antibodies: the past and the future in clinical application. J Transl Med 9 (2011).

20. Hodi FS, O'day SJ, Mcdermott DF et al. Improved survival with ipilimumab in patients with metastatic melanoma. The New England journal of medicine 363(8), 711-723 (2010).

21. Hamanishi J, Mandai M, Matsumura N, Abiko K, Baba T, Konishi I. PD-1/PD-L1 blockade in cancer treatment: perspectives and issues. International journal of clinical oncology 21(3), 462-473 (2016).

22. Mccoy KD, Le Gros G. The role of CTLA-4 in the regulation of T cell immune responses. Immunology and cell biology 77(1), 1-10 (1999).

23. Bakker E, Qattan M, Mutti L, Demonacos C, Krstic-Demonacos M. The role of microenvironment and immunity in drug response in leukemia. Biochimica et biophysica acta 1863(3), 414-426 (2016).

24. Linsley PS, Brady W, Grosmaire L, Aruffo A, Damle NK, Ledbetter JA. Binding of the B cell activation antigen $\mathrm{B} 7$ to $\mathrm{CD} 28$ costimulates $\mathrm{T}$ cell proliferation and interleukin 2 mRNA accumulation. The Journal of experimental medicine 173(3), 721-730 (1991).

25. Jago CB, Yates J, Camara NO, Lechler RI, Lombardi G. Differential expression of CTLA-4 among T cell subsets. Clinical and experimental immunology 136(3), 463-471 (2004).

26. Driessens G, Kline J, Gajewski TF. Costimulatory and coinhibitory receptors in anti-tumor immunity. Immunological reviews 229(1), 126-144 (2009).

27. Zou W, Chen L. Inhibitory B7-family molecules in the tumour microenvironment. Nature reviews. Immunology 8(6), 467-477 (2008).

28. Waterhouse P, Penninger JM, Timms E et al. Lymphoproliferative disorders with early lethality in mice deficient in Ctla-4. Science 270(5238), 985-988 (1995).

29. Tivol EA, Borriello F, Schweitzer AN, Lynch WP, Bluestone JA, Sharpe AH. Loss of CTLA-4 leads to massive lymphoproliferation and fatal multiorgan tissue destruction, revealing a critical negative regulatory role of CTLA-4. Immunity 3(5), 541-547 (1995).

30. Wu L, Yun Z, Tagawa T, Rey-Mcintyre K, De Perrot M. CTLA-4 blockade expands infiltrating T cells and inhibits cancer cell repopulation during the intervals of chemotherapy in murine mesothelioma. Molecular cancer therapeutics 11(8), 1809-1819 (2012). 
31. Tarhini AA. Tremelimumab: a review of development to date in solid tumors. Immunotherapy-Uk 5(3), 215-229 (2013).

32. Currie AJ, Prosser A, Mcdonnell A et al. Dual control of antitumor CD8 T cells through the programmed death-1/programmed death-ligand 1 pathway and immunosuppressive CD4 T cells: regulation and counterregulation. Journal of immunology (Baltimore, Md. : 1950) 183(12), 7898-7908 (2009).

33. Mansfield AS, Roden AC, Peikert T et al. B7-H1 expression in malignant pleural mesothelioma is associated with sarcomatoid histology and poor prognosis. Journal of thoracic oncology : official publication of the International Association for the Study of Lung Cancer 9(7), 1036-1040 (2014).

34. Combaz-Lair C, Galateau-Salle F, Mcleer-Florin A et al. Immune biomarkers PD-1/PD-L1 and TLR3 in malignant pleural mesotheliomas. Human pathology 52 9-18 (2016).

35. Khanna S, Thomas A, Abate-Daga D et al. Malignant Mesothelioma Effusions Are Infiltrated by CD3+ T Cells Highly Expressing PD-L1 and the PD-L1+ Tumor Cells within These Effusions Are Susceptible to ADCC by the Anti-PD-L1 Antibody Avelumab. Journal of thoracic oncology : official publication of the International Association for the Study of Lung Cancer 11(11), 1993-2005 (2016).

36. Sounni NE, Noel A. Targeting the tumor microenvironment for cancer therapy. Clinical chemistry 59(1), 85-93 (2013)

37. Finn OJ. Immuno-oncology: understanding the function and dysfunction of the immune system in cancer. Annals of oncology : official journal of the European Society for Medical Oncology / ESMO 23 Suppl 8 viii6-9 (2012).

38. Guo Y, Wang AY. Novel Immune Check-Point Regulators in Tolerance Maintenance. Frontiers in immunology 6421 (2015).

39. Salama AK, Hodi FS. Cytotoxic T-lymphocyte-associated antigen-4. Clin Cancer Res 17(14), 4622-4628 (2011).

40. Postow MA, Callahan MK, Wolchok JD. Immune Checkpoint Blockade in Cancer Therapy. Journal of Clinical Oncology 33(17), 1974-U1161 (2015).

41. Choe N, Tanaka S, Xia WJ, Hemenway DR, Roggli VL, Kagan E. Pleural macrophage recruitment and activation in asbestos-induced pleural injury. Environ Health Persp 105 1257-1260 (1997).

42. Anraku M, Cunningham KS, Yun Z et al. Impact of tumor-infiltrating T cells on survival in patients with malignant pleural mesothelioma. J Thorac Cardiov Sur 135(4), 823-829 (2008).

43. Hegmans JPJJ, Hemmes A, Hammad H, Boon L, Hoogsteden HC, Lambrecht BN. Mesothelioma environment comprises cytokines and T-regulatory cells that suppress immune responses. Eur Respir J 27(6), 1086-1095 (2006). 
44. Mutti L, Valle MT, Balbi B et al. Primary human mesothelioma cells express class II MHC, ICAM-1 and B7-2 and can present recall antigens to autologous blood lymphocytes. Int J Cancer 78(6), 740-749 (1998).

45. Sigalotti L, Coral S, Altomonte $\mathrm{M}$ et al. Cancer testis antigens expression in mesothelioma: role of DNA methylation and bioimmunotherapeutic implications. Brit J Cancer 86(6), 979-982 (2002).

46. Castagneto B, Zai S, Mutti L et al. Palliative and therapeutic activity of IL-2 immunotherapy in unresectable malignant pleural mesothelioma with pleural effusion - Results of a phase II study on 31 consecutive patients. Lung Cancer-J Iaslc 31(2-3), 303-310 (2001).

47. Valle MT, Porta C, Megiovanni AM et al. Transforming growth factor-beta released by PPD-presenting malignant mesothelioma cells inhibits interferon-gamma synthesis by an anti-PPD CD4+ T-cell clone. International journal of molecular medicine 11(2), 161-167 (2003).

48. Powell A, Creaney J, Broornfield S, Van Bruggen I, Robinson B. Recombinant GM-CSF plus autologous tumor cells as a vaccine for patients with mesothelioma. Lung Cancer-J Ias/c 52(2), 189-197 (2006).

49. Calabro L, Sigalotti L, Fonsatti E et al. Expression and Regulation of B7-H3 Immunoregulatory Receptor, in Human Mesothelial and Mesothelioma Cells: Immunotherapeutic Implications. J Cell Physiol 226(10), 2595-2600 (2011).

50. Calabro L, Morra A, Fonsatti E et al. Tremelimumab for patients with chemotherapy-resistant advanced malignant mesothelioma: an open-label, single-arm, phase 2 trial. The Lancet. Oncology 14(11), 1104-1111 (2013).

51. Nishino M GM, Suda M, Ramaiya Nh, Hodi Fs. Optimizing immune-related tumor response assessment: does reducing the number of lesions impact response assessment in melanoma patients treated with ipilimumab? J Immunother Cancer. doi:10.1186/2051-1426-2-17 (2014).

52. Wolchok JD, Hoos A, O'day S et al. Guidelines for the Evaluation of Immune Therapy Activity in Solid Tumors: Immune-Related Response Criteria. Clin Cancer Res 15(23), 7412-7420 (2009).

53. Nishino M. Immune-related response evaluations during immune-checkpoint inhibitor therapy: establishing a "common language" for the new arena of cancer treatment. J Immunother Cancer 4 (2016).

54. Weber JS, Hamid O, Chasalow SD et al. Ipilimumab Increases Activated T Cells and Enhances Humoral Immunity in Patients With Advanced Melanoma. J Immunother 35(1), 89-97 (2012).

55. Vonderheide RH, Lorusso PM, Khalil M et al. Tremelimumab in Combination with Exemestane in Patients with Advanced Breast Cancer and Treatment-Associated Modulation of Inducible Costimulator Expression on Patient T Cells. Clin Cancer Res 16(13), 3485-3494 (2010).

56. Calabro L, Morra A, Fonsatti E et al. Efficacy and safety of an intensified schedule of tremelimumab for chemotherapy-resistant malignant mesothelioma: an open-label, single-arm, phase 2 study. The Lancet. Respiratory medicine 3(4), 301-309 (2015). 
57. Astrazeneca. AstraZeneca reports top-line result of tremelimumab monotherapy trial in mesothelioma. (2016).

58. Kindler HL, Scherpereel A, Calabrò L et al. Tremelimumab as second- or third-line treatment of unresectable malignant mesothelioma (MM): Results from the global, double-blind, placebocontrolled DETERMINE study. 2016 ASCO Annual Meeting (2016).

59. Buchbinder El, Desai A. CTLA-4 and PD-1 Pathways: Similarities, Differences, and Implications of Their Inhibition. American journal of clinical oncology 39(1), 98-106 (2016).

60. Alley EW, Molife LR, Santoro A et al. Abstract CT103: Clinical safety and efficacy of pembrolizumab (MK-3475) in patients with malignant pleural mesothelioma: Preliminary results from KEYNOTE-028. Cancer Research 75(15 Supplement), CT103-CT103 (2015).

61. Wolchok JD, Kluger $\mathrm{H}$, Callahan MK et al. Nivolumab plus ipilimumab in advanced melanoma. The New England journal of medicine 369(2), 122-133 (2013).

62. Larkin J, Chiarion-Sileni V, Gonzalez R et al. Combined Nivolumab and Ipilimumab or Monotherapy in Untreated Melanoma. The New England journal of medicine 373(1), 23-34 (2015).

63. Morrissey KM, Yuraszeck TM, Li CC, Zhang Y, Kasichayanula S. Immunotherapy and Novel Combinations in Oncology: Current Landscape, Challenges, and Opportunities. Clinical and Translational Science 9(2), 89-104 (2016).

64. Calabrò L, Morra A, Giannarelli D et al. Tremelimumab and durvalumab (MEDI4736) combination for first and second-line treatment of mesothelioma patients: The NIBIT-MESO-1 study. 2016 ASCO Annual Meeting (2016).

65. Feig $\mathrm{C}$, Jones JO, Kraman $\mathrm{M}$ et al. Targeting CXCL12 from FAP-expressing carcinoma-associated fibroblasts synergizes with anti-PD-L1 immunotherapy in pancreatic cancer. Proceedings of the National Academy of Sciences of the United States of America 110(50), 20212-20217 (2013). 